\title{
LASER SCANNER RELIEFS OF SELECTED ARCHEOLOGICAL STRUCTURES IN THE SUBMERGED BAIAE (NAPLES)
}

\author{
B. Davidde Petriaggia ${ }^{a}$ G. Gomez de Ayala ${ }^{b}$ \\ astituto Superiore per la Conservazione ed il Restauro. barbara.davidde@beniculturali.it \\ $\underline{\text { bnaumacos@libero.it }}$
}

Commission V

KEY WORDS: Scuba Laser scanner documentation, Baiae, Underwater cultural heritage

\begin{abstract}
:
In 2011 the ISCR (Rome), in the frame of the Project Restoring Underwater directed by Barbara Davidde Petriaggi, started to test Naumacos Laser Scann 1, designed by Gabriele Gomez de Ayala, in order to document the restoration of a room paved with opus sectile probably part of the Bath of Punta Epitaffio (Underwater Park of Baiae - Marine Protected Area, Naples).

The experimentation conducted in Baiae by ISCR has shown the effectiveness of the Laser Scanner; this method also allowed to considerably reduce times and costs of underwater surveying. Moreover, the $3 \mathrm{D}$ relief obtained, has the characteristic of being geometrically (accuracy is sub-millimetric) and chromatically faithful to the reconstructed structure, as well as being exportable in various forms and usable in several contexts. From 2011 to 2013 the evolution of the instrument Naumacos Laser Scanner 3 was developed and tested in the restoration work of the Villa con ingresso a protiro, where three structures were documented in 3D (a paved with black and white mosaic decorated with hexagons and peltae, a very fragmentary black and white mosaic and a stone artefact. This paper shows the results of this documentation campaign and it underlines the prominent role in documentation and in museum display of Underwater Cultural Heritage played by the three-dimensional laser scanning survey. This technique also contributes to the increase of the value of scientific dissemination.
\end{abstract}

\section{INTRODUCTION}

The use of laser scanners for 3D acquisition is commonplace on dry land and is now also entering the field of underwater archaeology.

3D laser scanning delivers more precise results than 3D photogrammetry, but requires instruments that are still relatively costly. It uses a family of technologies, each of which has been tuned to meet the requirements of acquiring objects of different types, from the smallest, such as coins, to entire topographical areas or buildings. Scanners of this kind allow the rapid automatic acquisition of the position of thousands of points, known as point clouds. These define the surface of an object or a site, producing a 3D model that can be rendered for visualisation. The scanning pipeline consists of various phases, the first of which is the acquisition of the geometrical shape of the object, which can require scanning from many different directions. This is followed by alignment or registration, which allows the different scans to be merged automatically into a 3D model. Appropriate software, some of which is available free, is also required to process the data produced by the hardware.

The systems available to locate and map underwater archaeological sites (on and within the seabed) are essentially the multi-beam systems (sidescan sonar, sub-bottom profilers, magnetometers, and single and multibeam echosounders) and the interpretation of stereoscopic pictures.

The first group of tools produce a 3D graphic representation of the seabed morphology, particularly suitable to the geological study of the conformity of the seabeds and underwater canyons and to the localization of archaeological sites (wrecks, isolated items or archaeological structures); the second system produces a 3D model through the interpretation of stereoscopic pictures by means of particular software created for this kind of use (available freeware) that interprets the pictures in order to obtain a 3D model roughly like the original one (Canciani, Gambogi et alii 2002; Petriaggi, Davidde 2010, Mahon, Pizarro et alii 2011; Diamanti, Georgopoulos et alii 2011; Gearhart 2011).
In 2010/2011 the ISCR, in the frame of the project Restoring Underwater directed by Barbara Davidde Petriaggi (http://www.iscr.beniculturali.it/pagina.cfm?usz=1\&uid=69\&id pro=4), started a collaboration with Naumacos Underwater Archaeology (Gabriele Gomez de Ayala) to develop underwater three-dimensional scanning systems.

The experimentations were conducted in the Underwater Park of Baiae - Marine Protected Area, Naples. Here the ISCR is directing the project Restoring Underwater devoted to tests tools, materials and methods to restore and in situ protect underwater archaeological structures and underwater Cultural Heritage. Restoring underwater was designed in 2001 by Roberto Petriaggi who directed the restoration works in Torre Astura (Rome), Baiae and in other underwater archaeological sites in Italy (Petriaggi, 2004; Petriaggi, Mancinelli, 2004; Petriaggi 2005; Petriaggi, Davidde 2010; 2012; Petriaggi, Davidde Petriaggi, 2015). The ISCR Unit of underwater archaeology in the past years have focused its efforts to test 3D documentation systems, as the three-dimensional survey of the sarcophagi of the wreck off San Pietro in Bevagna (Taranto) using ScubaScan system by Menci Software (Petriaggi, Davidde 2010) or participating to the 3D documentation campaigns conducted with Università della Calabria (Gallo et alii 2012;. Bruno et alii, 2013)

The scanners developed in Baiae by Gabriele Gomez de Ayala and ISCR, object of this paper, produce, as we describe below, dense point clouds for the elaboration of 3D models with submillimetric resolution, simultaneously extrapolating from surface texture information and digital images. The equipment uses on-board GPS to orient the 3D images in order to assemble $3 \mathrm{D}$ composites of large areas, aligning the different individual scans in post-production using a system of optical targets.

In Italy another example of underwater laser scanner is tested by ENEA Frascati's Laser Vision Lab that is perfecting an underwater laser scanner called RE-VUE (Remote Viewing in Underwater Environments) which can be used at depths of up to 400 metres. (Further information and a bibliography is available at 
http://www.frascati.enea.it/utaprad/3D laser scanner sub.htm.) (BDP).

\section{THE ARCHAEOLOGICAL SITES}

The first season field (2011) was focused on the 3D scan of a room paved with opus sectile probably part of the Bath of Punta Epitaffio, near the Nymphaeum of the emperor Claudius in Baiae, dated between the early part of the first century $\mathrm{AD}$ and the third century AD. The opus sectile measures 3.5 metres by 4.5 metres and it consists of marble slabs of different shapes, sizes and grades.

The marble preserved only in the southwest corner, while for the remainder of the floor only the substrate of marble and brick fragments remains. The opus sectile reveals two forms of modulo semplice: one with large rectangular slabs and one of modulo a quadrato Q3. The former appears in the north section and at the edges while the central section has Q3. The principal geometrical layout is clearly visible, notwithstanding a number of bare patches. The central section contains triangles and squares set alongside each other and enclosed in a wide border of polychromatic slabs. A second narrower band of rectangular slabs runs round the whole composition. The internal western edge consists of a single row of rectangular slabs, whereas the short northern edge uses a triple row. The other two sides, where almost all of the flooring has been destroyed, probably mirrored the design of the two extant sides. Only three quadrants of the central area remain intact, each consisting of a square central slab encircled by four triangular pieces, one on each side, in the shape of a four-pointed star. Four further triangular pieces are positioned to complement the star to form a larger square. The marble used are Pavonazzetto, Pentelic, African, Docimio, Portasanta, white and Breccia di Aleppo marbles (Fig. 1) (Davidde Petriaggi et alii, in press).

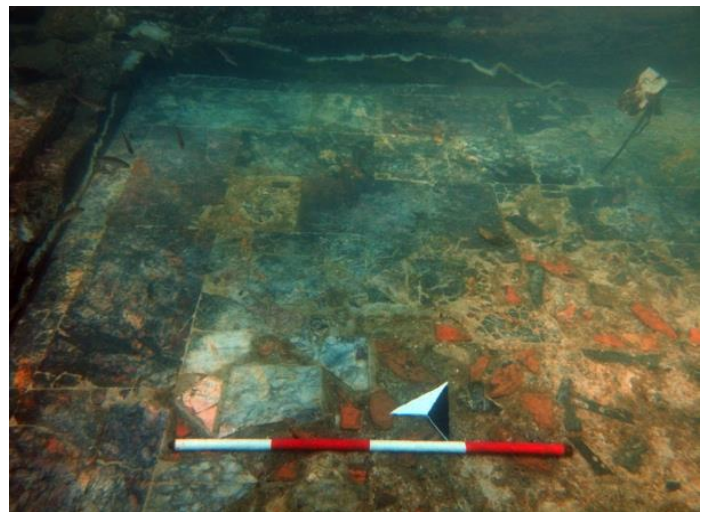

Fig. 1. Baiae. A particular of the opus sectile floor after restoration (ISCR Archive).

The second 3D documentation campaign was conducted in the Villa con ingresso a protiro.

This villa is one of the many villae maritimae along the lacus baianus. It has two clearly defined areas: the residential quarters and the baths. Between them lies a rectangular area probably a garden area. Here a statue, similar in style to the Aphrodite of the Gardens by Alcamenes, was recovered and is now housed in the Archaeological Museum of Campi Flegrei. The prothyrum was framed by a pair of columns and two long stone seats. The vestibule, with doorways to the ostiarius or gate-keeper's lodge, led to the atrium, whose walls were lined with marble panels, as were those of adjoining areas, many of which had mosaic flooring. A room at the north-east corner of the atrium with a mosaic of black and white tesserae with geometrical motifs (hexagons and pseudo-emblema with circles and peltae) was restored by ISCR and 3D documented using laser scanner (Fig.2). A large hall with a 10.37 metres wide apse lies to the south of the atrium. This was probably not part of the original design.

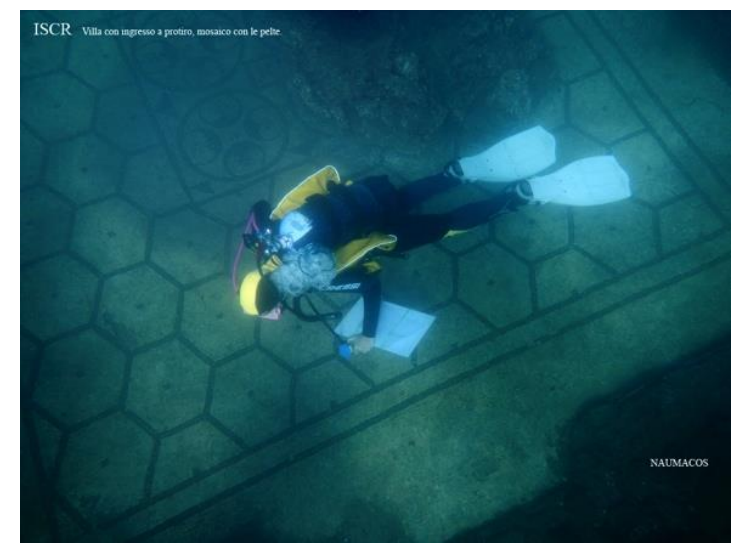

Fig. 2 - Villa con ingresso a Protiro, the black and white mosaic decorated with hexagons and peltae. (ISCR Archive)

This mosaic lies inside a rectangular room measuring 4.77 metres by 5.55 . The mosaic consists of a background of white tesserae with ornamental motifs created using a double line of black tesserae. The principal motif is a series of hexagons covering the whole floor, with a square of four black tesserae at the centre of each hexagon. Black tesserae also make up the double border around the edges. The central section is a pseudoemblema consisting of four circles enclosed by a large square, with four peltae inside each circle. Three equidistant solid black equilateral triangles lie between the circles inside each side of the square. There is a quincunx (the five-spot motif on six-sided dice) at the centre of each circle. The point of each pelta terminates in a small flower with three heart-shaped petals separated by a central dot. The remaining space between the four circles contains a small square enclosing a cruciform floral motif with a central dot. The floral motif has lobed petals with arrowhead tips. The mosaic dates back to the second century BC. Together with this mosaic, the 3D documentation involved also a very fragmentary black and white mosaic (Fig. 3 A) and a stone artefact probably a threshold of a sliding door (Fig. 3 B).

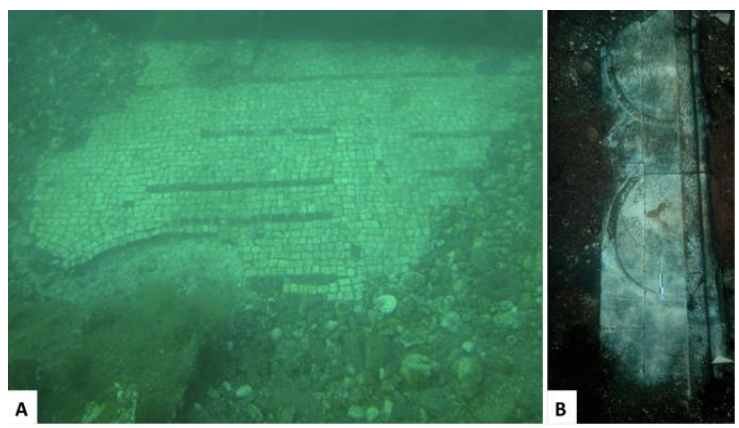

Fig. 3 - Villa con ingresso a protiro. A, a detail of black and white mosaic floor. B, threshold of a sliding door.

In particular, for these purposes, 3D relief has been very helpful to study the differential degradation of the mosaic tesserae and also to well document the shape and the mode of operation of a stone artefacts identified as a threshold of a sliding door (BDP). 


\section{THE 3D LASER SCANNER DOCUMENTATION}

The first laser scanner developed and used to document the opus sectile room was based on laser line and triangulation for the calculation of the point cloud (Fig. 4 - 6). The first version of the system was mounted on a frame (Fig. 7). The frame allowed to know the exact position of the scanner among the area to be scanned.

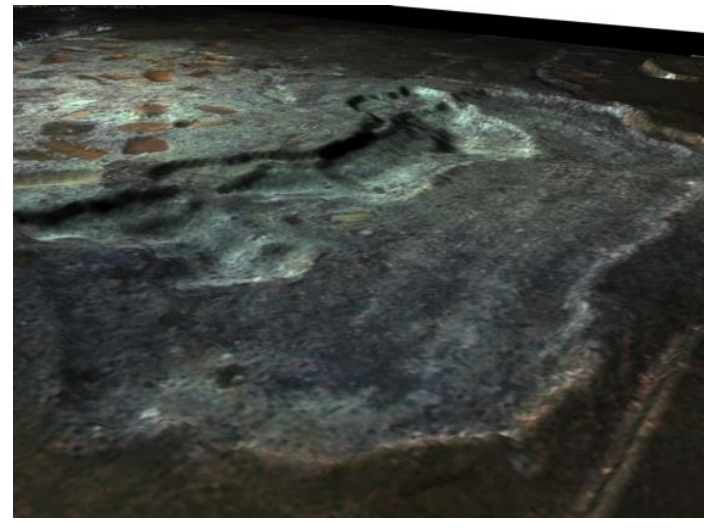

Fig. 4 - The opus sectile floor: 3D results (ISCR Archive)

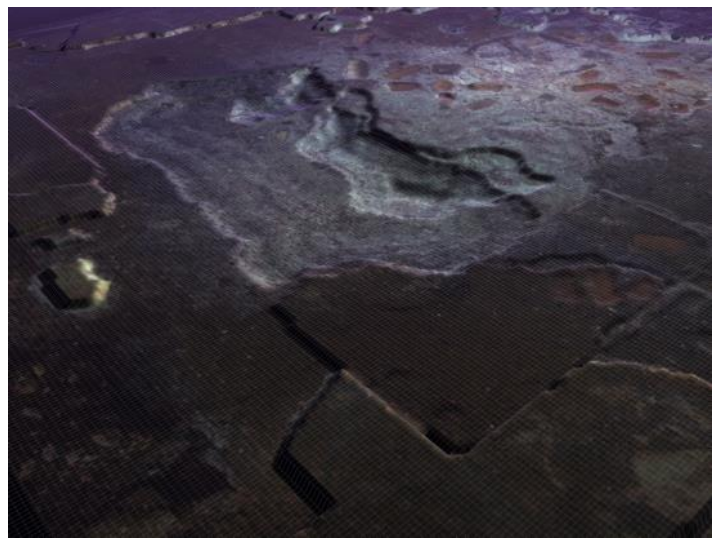

Fig. 5 - The opus sectile floor: 3D results (ISCR Archive)

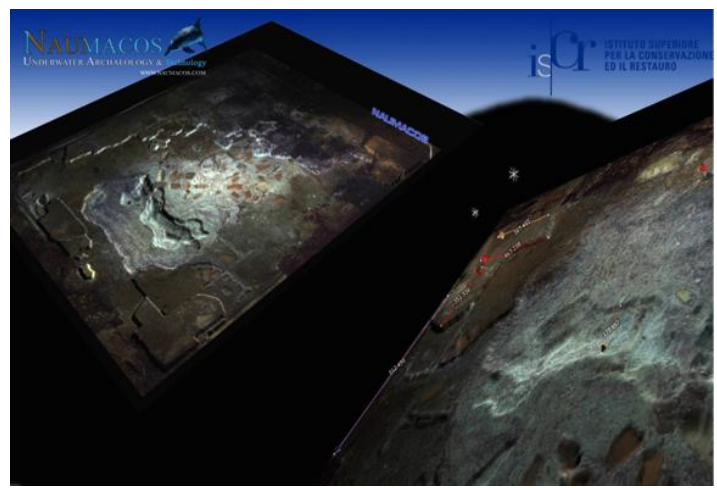

Fig. 6 - The opus sectile floor: 3D results (ISCR Archive)

In another phase of development, a portable scanning system was developed, based on infrared laser light structured. The system is operated by the diver simply framing the area to be scanned as a video camera. It can also be placed in front of the area to be scanned and activated, the system begins scanning rotating on its axis (Fig. 8).

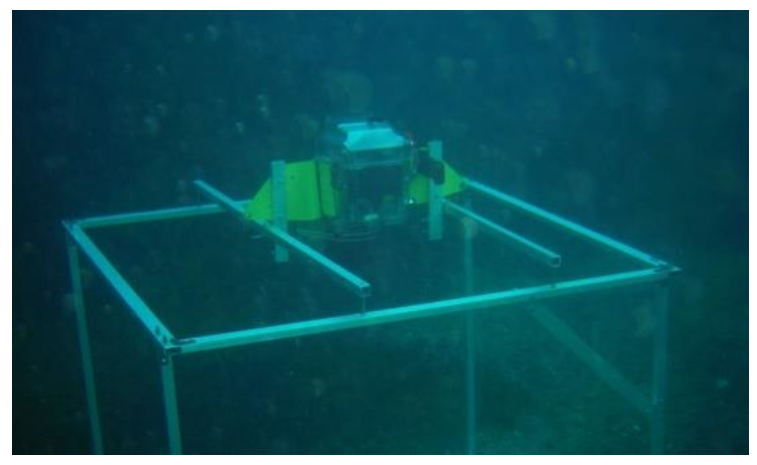

Fig. 7 - The first scanning system designed to scan at high resolution the erosion of the floor. (ISCR Archive)

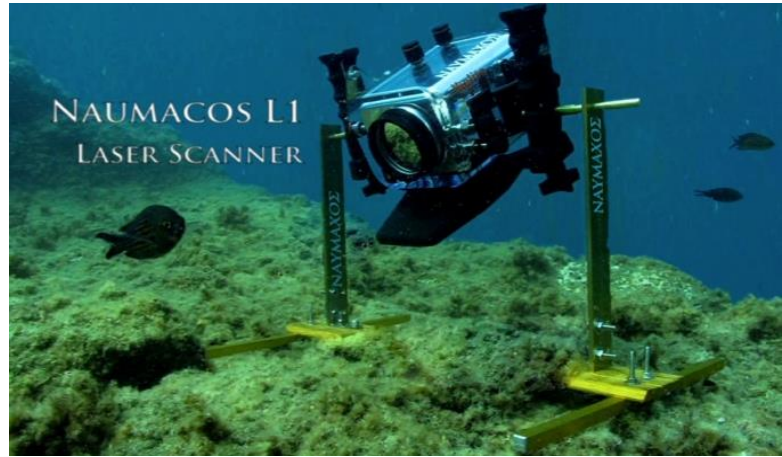

Fig. 8 - Portable scanning system, based on infrared laser light structured. When activated the system begins scanning rotating on its axis.

This light and compact instrument $(15 \times 16 \times 10 \mathrm{~cm}-4 \mathrm{~kg})$ was particularly valuable in producing an extremely precise and faithful 3D model of the original. To capture the level of detail required, the system was configured to give an accuracy of 0.05 $\mathrm{mm}$ at a resolution of 1,200,000 pixels. Many of the areas scanned were spatially aligned in post-production using reference points, shooting angles, solid forms and scanner metadata. The underwater scanner also captures textures which are subsequently rendered in the 3D model. These textures are acquired together with associated spatial coordinates, ensuring a perfectly faithful 3D model. Two different texture capture technologies are used, RGB point sampling and high-resolution spatially-annotated digital photography, which allows optimal results to be obtained in the variably turbid waters. For detailed scanning of larger areas, the system is configured to capture several square metres at a time, with an accuracy of $0.5 / 1 / 2 \mathrm{~mm}$ (Davidde, Petriaggi and Gomez De Ayala, 2014). A given area can be scanned from different angles with the results merged into a single $3 \mathrm{D}$ model later.

The upgraded version of the scanner used for the Villa con ingresso a protiro has offered a number of innovative advantages such as being able to capture surface detail in motion rather than from a fixed point (frame mounted) as was the case with the first one system.

The last system developed is based on structured light. When the light is projected on a surface it is deformed according to the shape of the surface, measuring the deformations it is possible to obtain three-dimensional data.

The system produces 30 scans per second and each scan is linked to the previous one. The accuracy depends on the needs, the system provides a centimetric to sub millimetric accuracy. The accuracy depends on the time of permanence of the system on the same place. Much more time on the same shot, the higher 
resolution of the result. The system produces points clouds and it is able to generate mesh in real time depending on whether it is configured with processors on board. It can scan continuously for 90 minutes covering a large area.

Contrariwise of the traditional terrestrial 3D scanner, this system can scan moving objects, it can also scan in motion itself, it doesn't need to be calibrated nor it needs to be placed any targets for its use. The operators are free to move while scanning the area of their interest. The result is a 3D model of all the surfaces sampled, and, at the same time a 3D motion capturing. By making a complete sampling of the site it will produce a three-dimensional digital map of the superficial layer and a detailed $0,05 \mathrm{~mm}$ mapping of the position of the Stratigraphic Unit. During the excavation or restoration work the scan of each layer records every single event and modification of the site and its condition. In the underwater environment it is not possible to take pictures from several meters because of the light absorption and for the low visibility through the water, the same is by scanning a surface of underwater objects so it is important to make several shootings from different angles. It is very important to line up each single scan accurately to avoid the possibility to add mistakes during this phase.

Follow a few photographs of the 3D survey carried out in the Villa con ingresso a protiro (Fig. 9 - 12). In this case, the accuracy is sub millimetric, because there is the need to recognize the individual mosaic tiles and monitor their erosion overtime (BDP, GDA).

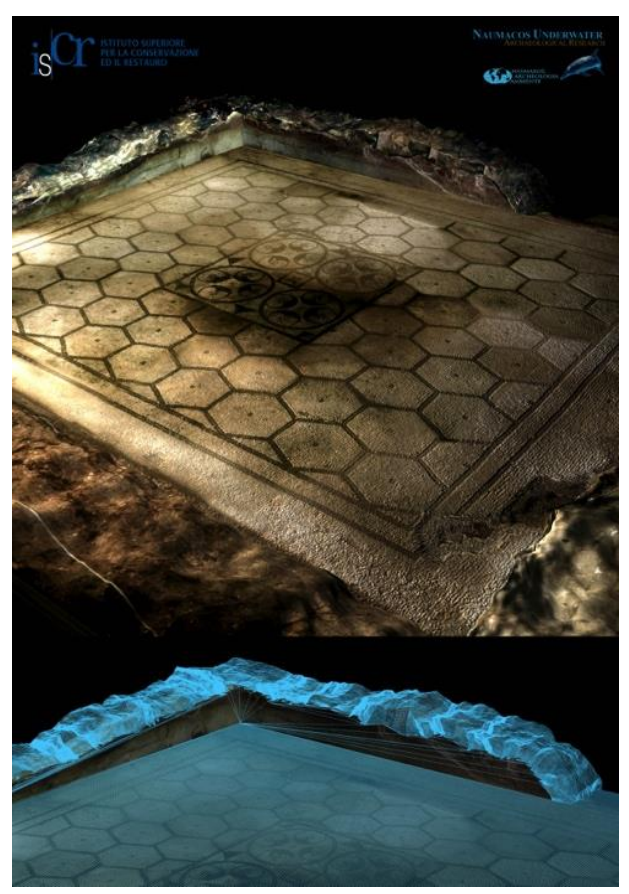

Fig. 9 - Laser Scanner relief of the bichrome mosaic with peltae. Villa con ingresso a protiro. (ISCR Archive)

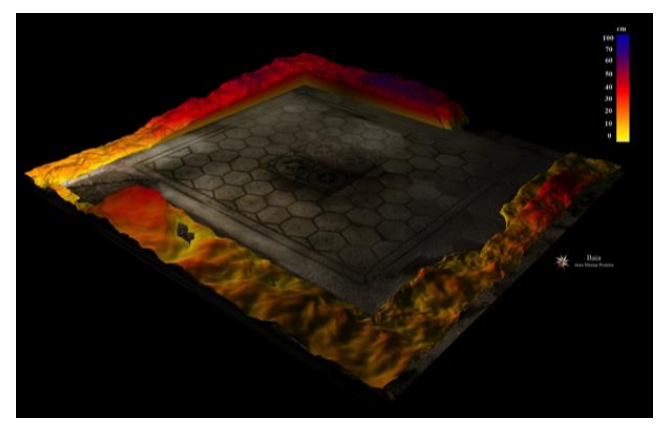

Fig. 10 - Laser scanner relief. Color progression from yellow to blue indicates different highs. (ISCR Archive)

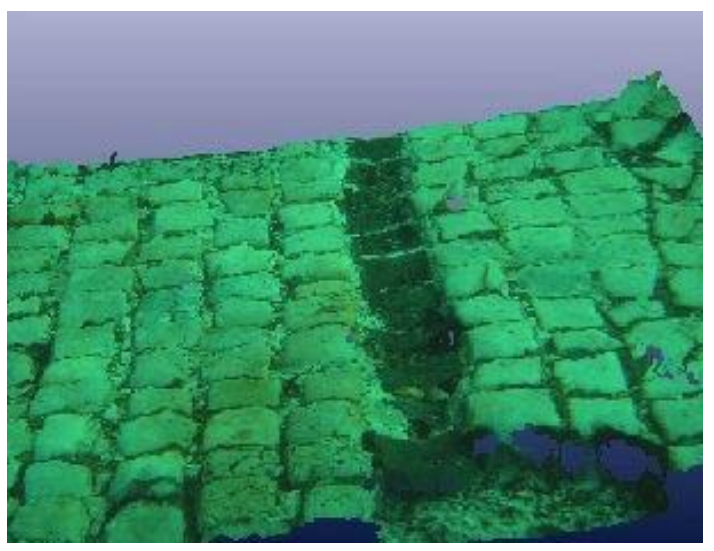

Fig. 11 - Laser scanner relief of the differential degradation of the black and white tesserae. (ISCR Archive)

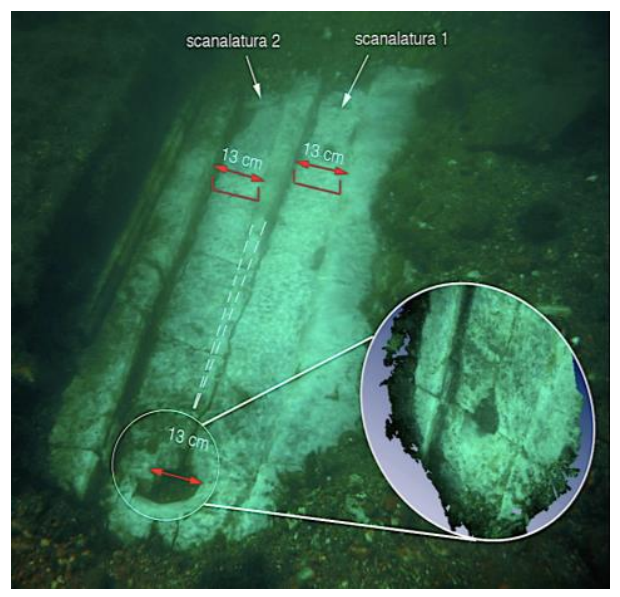

Fig. 12 - Treshold of a sliding door and its detailed laser scanner relief in the circle. (ISCR Archive)

\section{CONCLUSIONS}

The use of 3D scuba laser scanner has been useful to study, restore and document some selected underwater artefacts in Baiae (Petriaggi and Davidde Petriaggi 2015:221-223; 263264). This system produces an extremely precise and faithful 3D model of the original and for this reason it is particularly suitable for the in situ archaeological activities. These underwater sites have been an ideal setting for this system, since there are many details, small items, and a few undercuts, so they will need just few scans from different angles, and only sufficient zenith scans parallel will be necessary. 
This underwater scanner is an open system, well-suited to the unpredictably changeable conditions of underwater archaeological sites. This was invaluable in the production of archaeological documentation and the planning of conservation and maintenance tasks.

The three-dimensional laser scanning survey plays a prominent role in the documentation and museum display of Underwater Cultural Heritage, increasing the value of scientific dissemination (BDP).

\section{REFERENCES}

Bruno, F., Gallo, A., De Filippo, F., Muzzupappa, M., Davidde Petriaggi, B., Caputo, P., 2013. 3D documentation and monitoring of the experimental cleaning operations in the underwater archaeological site of Baia (Italy), Digital Heritage International Congress (Digital Heritage), vol.1, pp.105-112.

Canciani, M., Gambogi, P., Romano, G., Cannata, G. and Drap, P., 2002. Low cost digital photogrammetry for underwater archaeological site survey and artefact insertion. The case study of the Dolia Wreck in Secche della Meloria, Livorno, Italia. International Archives of Photogrammetry, Remote Sensing and Spatial Information Sciences 34.5 (W12), pp. 95-100.

Davidde Petriaggi, B., Petriaggi, R., Gomez de Ayala, G., 2014. 3D Documentation for the Assessment of Underwater Archaeological Remains. In CAA 2012 Proceedings of the 40th Conference in Computer Applications and Quantitative Methods in Archaeology, Southampton, United Kingdom, 26-30 March 2012, Archaeology in the Digital Era, Volume II, AMSTERDAM UNIVERSITY PRESS, pp. 174-180.

Davidde Petriaggi, B., Ricci, S., Poggi, D., 2014. The restoration in situ of a pavement in opus sectile in the Underwater Archaeological Park of Baiae (Naples, Italy), in: IKUWA V, Cartagena, October 15 - 19, Cartagena, in press.

Diamanti, E., Georgopoulos, A. and Vlachaki, F., 2011. Geometric documentation of underwater archaeological sites. In: Proceeding of the XXIII CIPA Symposium, Prague, Czech Republic, 12/16 September 2011, edited by Karel Pavelka, 1-8. Prague, Czech Technical University in Prague, Faculty of Civil Engineering in cooperation with CIPA.

Gallo, A., Angilica, A., Bianco, G., De Filippo, F., Muzzupappa, M., Davidde, B., Bruno, F., 2012. 3D Reconstruction and virtual exploration of submerged structures: a case study in the underwater archaeological site of Baia (Italy). In: Proceedings of VAST2012 - International Symposium on Virtual Reality, Archaeology and Cultural Heritage, 19-21 November 2012, Brighton, UK.

Gearhart, R., 2011. Archaeological interpretation of marine magnetic data. In: The Oxford handbook of Maritime archaeology, edited by A. Catsambis, B. Fordand and D. L. Hamilton, 90-113. Oxford: Oxford University Press.

Mahon, I., Pizarro, O., Johnson-Roberson, M., Friedman, A., Williams, S. B., Henderson, J. C., 2011. Reconstructing Pavlopetri: Mapping the World's Oldest Submerged Town using Stereo-vision. In: IEEE International Conference on Robotics and Automation Shanghai International Conference Center May 9-13, 2011, Shanghai, China, In: Robotics and
Automation, (ICRA) IEEE International Conference, Shanghai 2011, pp 2315-2321.

Petriaggi, R., 2004. Restauro subacqueo delle strutture sommerse della peschiera romana di Torre Astura. Lazio e Sabina, 2, pp. 273-276.

Petriaggi, R., 2005. Nuove esperienza di restauro conservativo nel Parco Sommerso di Baia. Archaeologia Maritima Mediterranea, 2, pp. 135-147.

Petriaggi, R., Davidde, B., 2005. The analytical Data Card of Underwater archaeological finds (Samas) for diagnosing the deterioration. Archaeologia Maritima Mediterranea, 2, pp. 161170.

Petriaggi, R., Davidde, B., 2010. The sarcophagi from the wreck of San Pietro in Bevagna (TA): The subject of new works by the Istituto Superiore per la Conservazione ed il Restauro. Archaeologia Maritima Mediterranea 7, pp. 131-37.

Petriaggi, R., Davidde, B., 2012. The ISCR Project "Restoring Underwater": an evaluation of the results after ten years from the beginning. "Conservation and Management of Archaeological Sites", 4th Conference on Preserving Archaeological Remains in Situ, 14, Paris 2012, pp. 192-199.

Petriaggi, R., Davidde Petriaggi, B., 2015. Archeologia sott'acqua. Teoria e pratica. Seconda Edizione aggiornata. Fabrizio Serra Editore, Pisa - Roma.

Petriaggi, R., Mancinelli, R., 2004. An experimental conservation treatment on the mosaic floor and perimeter walls of room n. 1 of the so-called «Villa con ingresso a protiro» in the underwater archaeological park of Baia (Naples). Archaeologia Maritima Mediterranea, 1, pp. 109-126. 